\title{
Manejo da lesão renal aguda: uma revisão narrativa
}

\author{
Management of acute kidney injury: a narrative review
}

Tratamiento de la lesión renal aguda: una revisión narrativa

Júlia Maria Moreira Silva ${ }^{1 *}$, Carolina Mauricio Carvalho de Castro ${ }^{2}$, Carolina Pismel Mora Castilho ${ }^{3}$, Estefane Ribeiro Melo ${ }^{4}$, Gabriela Vargas Carneiro ${ }^{5}$, Mayara Cristina Siqueira Faria ${ }^{5}$, Priscila de Souza Rezende ${ }^{6}$, Yury de Souza Guirelle ${ }^{7}$, Tarsila Rejane Maia Nascimento ${ }^{8}$.

\section{RESUMO}

Objetivo: Descrever fatores de risco, etiofisiopatologia e manejo da Lesão Renal Aguda (LRA) em pacientes internados em Unidade de Terapia Intensiva (UTI). Revisão bibliográfica: A LRA, que configura declínio da função renal súbita, afeta até $50 \%$ dos pacientes em estado crítico. Para seu diagnóstico, é importante analisar diurese, aumento da creatinina e ritmo de filtração glomerular, já que pequenas mudanças nestes fatores podem ocasionar diferenças significativas nos desfechos de pacientes em estado crítico, sendo imprescindível a detecção precoce da doença. As causas mais frequentes dessa patologia são a sepse, a hipovolemia e os agentes nefrotóxicos. Em UTI, a LRA pode se instalar quando associada a alguns fatores de risco tais como insuficiência pulmonar, hepática e cardíaca, choque hipovolêmico, disfunções isquêmicas, eventos obstrutivos e infecção disseminada, além do tempo de internação prolongado. Geralmente, o tratamento da causa subjacente acarreta a recuperação do paciente, porém, algumas vezes, a Terapia de Substituição Renal (TSR) é necessária. Considerações finais: Compreende-se, então, que a LRA é uma síndrome de falência renal com disfunção multifatorial e prejuízo a diversos órgãos. Por ser letal, frequente em pacientes em UTI e de difícil manejo, faz-se necessário o diagnóstico precoce e manejo imediato e adequado.

Palavras-chave: Lesão renal aguda, Choque séptico, Função renal, Prevenção.

\begin{abstract}
Objective: Describe the main risk factors, etiophysiopathology and management of Acute Kidney Injury (AKI) in patients admitted to the Intensive Care Unit (ICU). Bibliographic review: AKI, which represents a sudden decline in renal function, affects up to $50 \%$ of critically ill patients. For its diagnosis, it is important to analyze diuresis, increase in creatinine and glomerular filtration rate, since slight changes in these factors can cause significant differences in the outcomes of critically ill patients, and early detection of the disease is essential. The most frequent causes of this pathology are sepsis, hypovolemia and nephrotoxic agents. Among the main risk factors for the development of AKI in the ICU are: ischemic, nephrotoxic, infectious, obstructive events, arterial hypotension, shock, cardiovascular, hepatic and respiratory insufficiencies, neoplasms and the average length of stay in the unit greater than seven days. Treatment of the underlying cause usually results in the patient's recovery, however, Renal Replacement Therapy (RRT) is sometimes necessary. Final considerations: It is understood that AKI is a syndrome of renal failure with multifactorial dysfunction and damage to several organs. Because it is lethal, frequent in ICU patients and difficult to manage, an early diagnosis and immediate and appropriate management are necessary.
\end{abstract}

Keywords: Acute kidney injury, Septic shock, Kidney function, Prevention.

\footnotetext{
${ }^{1}$ Centro Universitário do Planalto Central Apparecido dos Santos (Uniceplac), Gama - DF.

*E-mail: juliamoreira.ms@gmail.com

2 Universidade Nove de Julho (UNINOVE), Bauru - SP.

${ }^{3}$ Centro Universitário UniCesumar (UniCesumar), Maringá - PR.

4 Universidade de Pernambuco (UPE), Garanhuns - PE.

${ }^{5}$ Centro Universitário São Lucas (UNISL), Porto Velho - RO.

6 Universidade Vila Velha (UVV), Vila Velha - ES.

7 Faculdade de Ensino Superior da Amazônia Reunida (FESAR), Redenção - PA.

8 Universidade Federal do Maranhão (UFM), São Luís - MA.
} 


\section{RESUMEN}

Objetivo: Describir los factores de riesgo, etiofisiopatología y manejo de la Lesión Renal Aguda (IRA) en pacientes ingresados en la Unidad de Cuidados Intensivos (UCI). Revisión bibliográfica: La LRA, que representa un descenso brusco de la función renal, afecta hasta al $50 \%$ de los pacientes críticos. Para su diagnóstico, es importante analizar la diuresis, el aumento de creatinina y el filtrado glomerular, ya que pequeños cambios en estos factores pueden provocar diferencias significativas en la evolución de los pacientes críticos, y la detección precoz de la enfermedad es fundamental. Las causas más frecuentes de esta patología son la sepsis, la hipovolemia y los agentes nefrotóxicos. Entre los principales factores de riesgo para el desarrollo de LRA en la $\mathrm{UCl}$ se encuentran: eventos isquémicos, nefrotóxicos, infecciosos, obstructivos, hipotensión arterial, shock, insuficiencia cardiovascular, hepática y respiratoria, neoplasias y la estadía promedio en la unidad mayor a siete días. El tratamiento de la causa subyacente generalmente da como resultado la recuperación del paciente; sin embargo, a veces es necesaria la Terapia de Reemplazo Renal (TRR). Consideraciones Finales: Se entiende, entonces, que la LRA es un síndrome de insuficiencia renal con disfunción multifactorial y daño a varios órganos. Por ser letal, frecuente en UCl y difícil de manejar, es necesario un diagnóstico precoz y un manejo inmediato y adecuado.

Palabras clave: Lesión renal aguda, Choque séptico, Función del riñón, Preventión.

\section{INTRODUÇÃO}

A Lesão Renal Aguda (LRA) é descrita, de acordo com os critérios do Kidney Disease Improving Global Outcomes (KDIGO), como o declínio da função renal súbita causando acúmulo de substâncias nitrogenadas (ureia e creatinina). Consiste em uma síndrome clínica com complicações do curso e piora do desfecho em muitos pacientes hospitalizados, sendo que dificilmente possui uma única fisiopatologia (MAKRIS $\mathrm{K}$ e SPANOU L, 2016). É válido ressaltar que alguns fatores se destacam como predisponentes para a LRA, dentre eles estão: doenças clínicas pré-existentes, intervenções terapêuticas e a predisposição individual e multifatorial, como envelhecimento e presença de outras comorbidades renais (BENICHEL C, et al., 2020).

A LRA afeta uma parcela significativa dos pacientes em estado crítico. Em UTI, a LRA pode se instalar quando associada a alguns fatores de risco, como insuficiência pulmonar, hepática e cardíaca, choque hipovolêmico, disfunções isquêmicas, eventos obstrutivos e infecção disseminada, além do tempo de internação prolongado. Uma importante preocupação em pacientes que desenvolvem LRA é que, mesmo após tratada, ela colabora para o aumento do período de hospitalização, potencializa a gravidade do quadro clínico e pode levar ao surgimento de novas doenças (AMORIM F, et al., 2017).

Em países desenvolvidos, estima-se que a incidência de LRA tende a ser maior. Entre os pacientes hospitalizados, a taxa de ocorrência chega a 15\%, sendo mais comum em pacientes graves, com até $60 \%$ dos pacientes da UTI acometidos. Alguns estudos têm como alvo populações especiais (idosos e crianças), entretanto, existem poucos estudos epidemiológicos na população infantil (MAKRIS K e SPANOU L, 2016).

Muitos pacientes com LRA são assintomáticos ou apresentam sintomas inespecíficos como náuseas, vômitos, fadiga, anorexia e prurido. Porém o quadro clínico costuma se apresentar tardiamente, e muitas vezes a condição só é suspeitada e diagnosticada através de exames laboratoriais (BENICHEL C, et al., 2020). Assim, torna-se crucial a prevenção da LRA e a identificação dos fatores de risco (BELLOMO R, et al., 2017)

A identificação da causa da LRA é essencial para o sucesso do tratamento, que é baseado na correção da causa de base e suporte clínico ao paciente (JOANNIDIS M, ET AL., 2017). Uma das causas mais comuns da LRA é o choque séptico e faz-se necessário o uso de terapia antimicrobiana precoce associada a ressuscitação volêmica para atingir estabilidade hemodinâmica do paciente (BELLOMO R, et al., 2017). Na maioria dos casos, tratar a causa de base leva a uma recuperação completa do quandro clínico, porém há casos em que é necessária terapia de substituição renal (SOLíS GR, et al., 2018).

A mortalidade na LRA é diretamente proporcional a sua gravidade. Uma lenta recuperação da função renal e uso indiscriminado de drogas vasoativas estão fortemente relacionados ao aumento da mortalidade, porém, uma queda para um estágio inferior dos critérios de AKIN (Acute Kidney Injury Network) em 7 dias está 
relacionado a uma maior sobrevivência. Entretanto, AKIN estágio 3 está associado a alta mortalidade mesmo que haja recuperação renal total em até 7 dias da admissão na unidade de terapia intensiva. (SANTOS RP, et al., 2020).

Este artigo tem como objetivo descrever os principais fatores de risco, etiologia, fisiopatologia e manejo da LRA, bem como evidenciar as consequências desta patologia no desfecho dos pacientes críticos internados em UTI.

\section{REVISÃO BIBLIOGRÁFICA}

Os rins são órgãos fundamentais ao pleno funcionamento do organismo. A capacidade de depuração é a função renal primordial. Além disso, os rins têm participação metabólica, endócrina e no equilíbrio hidroeletrolítico e acidobásico. A LRA consiste na diminuição abrupta da função renal, resultando em acúmulo das escórias nitrogenadas (ureia e creatinina), podendo apresentar ou não diminuição da diurese. De acordo com o Kidney Disease (KDIGO) mais recente, a LRA pode ser definida pela fusão dos critérios de RIFLE (Risk, Injury, Failure, Loss, End-Stage Renal Disease) e AKIN (Acute Kidney Injury Network). Eles levam em consideração a diurese, o aumento da creatinina e o ritmo de filtração glomerular. Pequenas mudanças na função renal de pacientes criticamente enfermos estão relacionadas a mudanças significativas nos desfechos destes pacientes (HUANG CY, et al., 2020; MAKRIS K e SPANOU L, 2016).

A definição mais aceita atualmente é a do KDIGO, sendo a LRA definida pelo aumento da creatinina sérica em $0,3 \mathrm{mg} / \mathrm{dL}$ ou mais, em até 48 horas, ou aumento dos níveis em pelo menos $50 \%$ do valor da creatinina de referência nos últimos 7 dias, ou diurese menor que $0,5 \mathrm{~mL} / \mathrm{kg} /$ hora durante 6 horas (AZEVEDO LCP, et al., 2020). O quadro abaixo mostra as principais classificações da LRA (Tabela 1).

Quadro 1 - Classificações de LRA.

\begin{tabular}{|c|c|c|c|}
\hline RIFLE & AKIN & KDIGO & DÉBITO URINÁRIO \\
\hline $\begin{array}{c}\text { Risco } \\
\uparrow \mathrm{Cr}>1,5 \mathrm{x} \text { o basal } \\
\downarrow \mathrm{TFG}>25 \%\end{array}$ & $\begin{array}{c}\text { Estágio 1 } \\
\uparrow \mathrm{Cr} \geq 0,3 \mathrm{mg} / \mathrm{dL} \\
\uparrow \mathrm{Cr}>1,5-2 \times \text { o basal }\end{array}$ & $\begin{array}{c}\text { Estágio 1 } \\
\uparrow \mathrm{Cr} \geq 0,3 \mathrm{mg} / \mathrm{dL} \\
\uparrow \mathrm{Cr}>1,5-1,9 \times \circ \text { basal }\end{array}$ & $\begin{array}{c}<0,5 \mathrm{~mL} / \mathrm{kg} / \text { hora por mais } \\
\text { que } 6 \text { horas }\end{array}$ \\
\hline $\begin{array}{c}\text { Lesão } \\
\uparrow \mathrm{Cr}>2 \mathrm{x} \circ \text { basal } \\
\downarrow \mathrm{TFG}>50 \%\end{array}$ & $\begin{array}{c}\text { Estágio } 2 \\
\uparrow \mathrm{Cr}>2 \text { - } 3 x \text { o basal }\end{array}$ & $\begin{array}{c}\text { Estágio } 2 \\
\uparrow \mathrm{Cr}>2-2,9 \mathrm{x} \text { o basal }\end{array}$ & $\begin{array}{c}<0,5 \mathrm{~mL} / \mathrm{kg} / \mathrm{h} \text { hora por mais } \\
\text { que } 12 \text { horas }\end{array}$ \\
\hline $\begin{array}{c}\text { Falência } \\
\uparrow \mathrm{Cr}>3 x \text { o basal OU } \\
\mathrm{Cr}>4 \mathrm{mg} / \mathrm{dL} \text { com } \uparrow \text { aguda } \\
>0,5 \mathrm{mg} / \mathrm{dL} \text { OU } \downarrow \text { TFG }> \\
75 \%\end{array}$ & \begin{tabular}{|c|} 
Estágio 3 \\
$\uparrow \mathrm{Cr}>3 \times$ × basal \\
$\mathrm{Cr}>4 \mathrm{mg} / \mathrm{dL}$ com $\uparrow$ aguda \\
$>0,5 \mathrm{mg} / \mathrm{dL}$ \\
$\mathrm{TSR}$
\end{tabular} & $\begin{array}{c}\text { Estágio } 3 \\
\uparrow \mathrm{Cr}>3 \times \text { o basal } \\
\mathrm{Cr}>4 \mathrm{mg} / \mathrm{dL} \\
\mathrm{TSR}\end{array}$ & $\begin{array}{c}<0,3 \mathrm{~mL} / \mathrm{kg} / \mathrm{hora} \text { por mais } \\
\text { que } 24 \text { horas OU anúria } \\
\text { por } 12 \text { horas }\end{array}$ \\
\hline $\begin{array}{l}\text { Perda da função } \\
\text { Perda completa da função } \\
\text { renal > } 4 \text { semanas }\end{array}$ & & & \\
\hline $\begin{array}{c}\text { Terminal } \\
\text { Doença renal em estágio } \\
\text { terminal por }>3 \text { meses }\end{array}$ & & & \\
\hline
\end{tabular}

Legenda: $\mathrm{Cr}=$ Creatinina; TFG = Taxa de filtração glomerular; TSR = Terapia de substituição renal Fonte: Silva JMM, et al., 2021.

O aumento da creatinina sérica ocorre na hipoperfusão renal mesmo com os rins estruturalmente intactos. No entanto, o nível de creatinina sérica é considerado um "padrão ouro imperfeito" no diagnóstico da LRA por ser insensível a mudanças agudas da função renal, ou seja, é marcadora da taxa de filtração glomerular e não diretamente do dano tubular (MAKRIS K e SPANOU L, 2016).

O débito urinário e a creatinina estão cada vez mais sendo complementados por novos biomarcadores de LRA (BELLOMO R, et al., 2017; SANTOS RP, et al., 2019). Esses biomarcadores aparecem logo no início da injúria renal, perduram durante o curso da doença e sua identificação permite o diagnóstico precoce de injúria renal, facilitando o tratamento, e contribuindo para um melhor prognóstico do paciente (THONGPRAYOON C, et al., 2020). 
Nesse sentido, os biomarcadores mais assertivos para a investigação da injúria renal são a cistatina C, uma proteína de pequena produção, cuja concentração em amostras sanguíneas maior que 0,91mg/L já chama atenção para lesão renal; e a lipocalina associada à gelatinase neutrofílica, a qual é uma proteína produzida no nefrón distal e, por estar associada aos neutrófilos, tem relação direta com inflamações no trato renal e sua presença, mesmo que pequenas quantidades acima de $9,8 \mathrm{ng} / \mathrm{ml}$ pode indicar injúria renal. Além desses, estão em fase de estudos outros biomarcadores, tais como a interleucina 1 (IL-1) e a molécula de injúria renal 1 (KIM-1) (THONGPRAYOON C, et al., 2020).

Há, também, estudos de validação que devem utilizar análise de calibração e de curva decisória, além das análises de discriminação, recomenda-se o uso dos critérios KDIGO para lesão renal aguda e as definições estabelecidas para definir o valor basal de creatinina sérica (HUANG CY, et al., 2020).

Várias evidências apontam que a LRA não consiste apenas na falência renal, isoladamente, mas sim em uma síndrome na qual a disfunção do rim desencadeia ativamente o desenvolvimento de disfunções em outros órgãos, através de mecanismos pró-inflamatórios envolvendo a migração de neutrófilos, liberação de citocinas (interleucina 1, interleucina 6, interleucina 10 e fator de necrose tumoral alfa) e amplificação do estresse oxidativo (MAKRIS K e SPANOU L, 2016).

Desse modo, ela pode afetar os sistemas respiratório, cardíaco, nervoso e imunológico, ao causar, por exemplo, edema, lesão aguda, arritmia, insuficiência cardíaca congestiva, encefalopatia urêmica, demência e disfunção sistêmica. Assim, a não recuperação da função renal pode acarretar sérias complicações hemodinâmicas, imunológicas e humorais (SANTOS RP, et al., 2020).

A fisiopatologia da LRA é extensa e ainda não totalmente elucidada. Sabe-se que os mecanismos fisiopatológicos envolvidos podem ser decorrentes de causas pré-renais (hipovolemias, diminuição do débito cardíaco, vasodilatação periférica, vasoconstrição renal e drogas), renais (doenças glomerulares e vasculares, nefrite intersticial aguda, nefrotoxinas) e pós-renais (obstrução bilateral dos ureteres, obstrução em bexiga e na uretra) (AMORIN F, et al., 2017).

Dentre as principais causas de LRA, o choque séptico está em primeiro lugar (LUFT J, et al., 2016), visto que nele a circulação passa a ser hiperdinâmica, diminuindo rapidamente a taxa de filtração glomerular (MAKRIS K e SPANOU L, 2016). Com isso, ocorre uma série de eventos em cadeia: alterações hemodinâmicas renais, disfunção endotelial, infiltração de substâncias inflamatórias no parênquima renal, trombose e necrose (COSTA NA, et al., 2020).

As substâncias inflamatórias possuem papel essencial na fisiopatologia da LRA associada a sepse. A resposta inflamatória desregulada que ocorre durante a sepse leva à liberação de citocinas e quimiocinas, ativa o sistema complemento, aumenta a produção de radicais livres de oxigênio e metabólitos do ácido araquidônico, ativa fatores extrínsecos de coagulação e células $T$ e recrutam neutrófilos, macrófagos, plaquetas e células endoteliais. Esses fatores têm efeito tóxico nas células tubulares causando danos na superfície luminal, podendo até mesmo induzir a apoptose das células renais (BELLOMO R, et al., 2017; FANI F, et al., 2017).

Ademais, a disfunção endotelial está associada à perda de vasodilatação mediada por óxido nítrico (NO), levando ao aumento das respostas vasoconstritoras que levam a diminuição da perfusão e, por fim, hipóxia tecidual (BELLOMO R, et al., 2017; FANI F, et al., 2017). Assim, a LRA séptica possui um pior prognóstico em relação a não séptica (MAKRIS K e SPANOU L, 2016; PINHEIRO KHE, et al., 2019; YOUNES-IBRAHIM $M, 2020)$.

O desequilíbrio dos fluidos corporais gera edema intersticial renal e retenção de água e solução salina, ocasionando um aumento da pressão intersticial, redução do fluxo sanguíneo renal e taxa de filtração glomerular, aumento da pressão intra-abdominal, pressão venosa central e pressão venosa renal, o que leva a piora da função renal. $\mathrm{O}$ uso de diuréticos minimiza as consequências da sobrecarga hídrica (MAKRIS $\mathrm{K}$ e SPANOU L, 2016; PINHEIRO KHE, et al., 2019; YOUNES-IBRAHIM M, 2020). 
Cerca de $20 \%$ dos pacientes sépticos não graves desenvolvem LRA, subindo para 35 a $65 \%$ no caso de pacientes sépticos críticos. Em média $50 \%$ dos pacientes em terapia intensiva com LRA vão a óbito, sendo que os pacientes que sobrevivem possuem um grande risco de posteriormente apresentarem doença renal crônica (PETERS E, et al., 2018).

A maior gravidade da LRA está relacionada ao aumento da mortalidade, porém a queda para um estágio inferior do critério de AKIN em 7 dias está associada ao aumento da sobrevivência. No entanto, no AKIN estágio 3 há alta mortalidade mesmo com recuperação renal completa dentro de 7 dias da admissão na Unidade de Terapia Intensiva (UTI) (PETERS E, et al., 2018). A não recuperação da função renal após LRA e o uso indiscriminado de drogas vasoativas durante internação na UTI são os principais fatores associados à mortalidade dos pacientes. Sendo assim, é necessária a busca ativa por fatores que agravam ou mantêm o episódio de LRA (SANTOS RP, et al., 2020).

Além da sepse, uma condição importante pode desencadear a LRA: a hipovolemia, que pode ser causada por hemorragias, perdas gastrointestinais ou cutâneas etc. $O$ aporte sanguíneo insuficiente pode levar a diminuição da perfusão, sendo um potencial causador da hipóxia. Nesse caso, a ressuscitação com fluidos objetiva alcançar a preservação do volume sanguíneo circulatório eficaz, recuperando a perfusão tecidual ao ponto necessário para manter uma adequada captação de oxigênio. A monitorização da Pressão Venosa Central (PVC), Pressão Arterial Média (PAM) e Saturação de Oxigênio $\left(\mathrm{SvO}_{2}\right)$ colaboram para avaliação da volemia (JOANNIDIS M, et al., 2017).

Outra causa de LRA é a rabdomiólise, condição causada por dano muscular extenso, podendo ter sido ocasionado por trauma muscular, hipertermia, exercício físico extenuante, exposição a determinadas drogas etc. Esse dano muscular promove a liberação de eletrólitos, mioglobinas, proteínas que, em determinado grau, podem ser nefrotóxicos, desencadeando a cascata inflamatória já mencionada (MAKRIS K e SPANOU L, 2016; THONGPRAYOON C, et al., 2020).

Dentre os diagnósticos feitos na admissão hospitalar, o que mais se associa a LRA são doenças respiratórias associadas às dependências ventilatórias (BENICHEL C, et al., 2020). Diante disso, a Ventilação Mecânica Invasiva (VMI) é amplamente utilizada em ambientes de UTI, principalmente em casos de insuficiência respiratória aguda ou crônica agudizada. Em contrapartida, esse recurso mostrou-se como importante fator contributivo para o agravo da função renal em diferentes gradações. Constatou-se que pacientes em VMI com Pressão Positiva Expiratória Final (PEEP) $>5 \mathrm{cmH} 2 \mathrm{O}$ estão mais predispostos ao aparecimento da LRA em UTI, em razão da diminuição da perfusão renal causada pela redução do débito cardíaco, além da tendência à idade avançada, sobrepeso, tempo prolongado de ventilação mecânica e ainda hipertensão arterial (SILVA KGN, et al., 2020).

O quadro clínico da LRA costuma ser protraído, com muitos pacientes assintomáticos. É comum que a LRA seja suspeita ou diagnosticada a partir, apenas, da análise dos exames laboratoriais. Sendo assim, fica mais uma vez evidente a importância da monitorização frequente da função renal no diagnóstico precoce dessa patologia. As manifestações clínicas, quando aparecem, costumam ser tardias, quando a lesão já está instalada e grave. Alguns pacientes apresentam sinais e sintomas diretamente relacionados aos rins, como hematúria. Também há relatos de anorexia, fadiga, náuseas e vômitos e prurido (BENICHEL C, et al., 2020).

Algumas informações são cruciais para a prevenção e o tratamento da LRA. A prioridade a princípio é a prevenção, identificando os pacientes com risco aumentado. A identificação de risco é feita principalmente por dois parâmetros: pacientes adultos criticamente enfermos com suscetibilidades conhecidas (idade, diabetes mellitus, insuficiência cardíaca, doença renal crônica, doença hepática) e outros fatores eventualmente modificáveis (produção de urina, balanço de fluidos) (BELLOMO R, et al., 2017).

Após instalada a LRA, a identificação de sua etiologia é imprescindível para o sucesso do tratamento, visto que a correção do fator desencadeante é básica e primordial, além de suporte clínico para estabilização do quadro. Pode-se alcançar a melhora ou até preservação da perfusão renal através do aumento do débito cardíaco por meio da ressuscitação com fluidos ou drogas inotrópicas, pelo uso de vasopressores sistêmicos ou vasodilatadores renais (JOANNIDIS M, et al., 2017). 
As drogas vasoativas continuam sendo a base do manejo da hipotensão e podem restaurar a pressão de perfusão adequada do órgão. As drogas vasoativas mais comumente utilizadas são a noradrenalina, adrenalina, vasopressina, dopamina e fenilefrina (BELLOMO R, et al., 2017). Se comparada com a dopamina, a norepinefrina aplicada como vasopressor inicial está associada a menos taquicardia durante as primeiras horas, sendo superior no tocante à sobrevida em pacientes com choque cardiogênico (JOANNIDIS M, et al., 2017).

A reposição volêmica pode ocorrer por uso de cristaloides isotônicos, que consistem no principal suporte na correção da depleção do volume extracelular, devendo ter cautela para evitar a hipercloremia e reduzir a potencial vasoconstrição renal. Além da reposição volêmica por cristaloides, há também o uso de coloides ou suas combinações. Porém, a reposição de grandes volumes com o uso dos coloides isoladamente possui risco de comprometimento hiperoncótico da filtração glomerular e consequente dano osmótico tubular. Entre os colóides artificiais disponíveis, há a gelatina, dextranos e amidos, sendo que a gelatina possui efeito moderado no volume, apesar de apresentar risco de nefrose osmótica (JOANNIDIS M, et al., 2017).

Já os dextranos possuem efeitos satisfatórios no volume, porém o uso de doses acima de 1,5 g/kg/dia pode predispor ocorrência de LRA, distúrbios de coagulação, anafilaxia e nefrose osmótica. A albumina humana apresenta-se como um coloide natural de uso eficaz e seguro na hipovolemia, visto que ela proporciona aumentos na resposta aos diuréticos nos pacientes com hipoalbuminemia, além de não apresentar efeitos negativos na função renal (JOANNIDIS M, et al., 2017).

A sobrecarga hídrica está relacionada com a LRA, podendo também ser causa ou consequência da lesão e, por isso, é tida como um possível marcador prognóstico da LRA, tendo em vista que após a ocorrência da sobrecarga hídrica há o aumento da creatinina e diminuição da diurese. Ainda assim, existem pacientes em que ocorre a hemodiluição pela sobrecarga de fluidos e consequentemente apresentam creatinina a um nível subestimado, restringindo a identificação da LRA por meio dos atuais critérios de diagnósticos (SANTOS RP, et al., 2020).

Quando a LRA é causada pelo seu fator mais comum, o choque séptico, tratar esta causa se mostra fundamental para obter êxito no quadro como um todo. Entre os tratamentos do choque séptico, tem-se a ressuscitação hemodinâmica e respiratória precoce e tratamentos com antibióticos apropriados. Segundo Bellomo R (2017), a terapia antimicrobiana precoce e apropriada, junto ao controle da fonte séptica, foi associada a um menor risco de LRA (ANNANE DJILLALI MD, et al., 2018).

Para cada hora em que a antibioticoterapia apropriada foi adiada, o risco de LRA aumentou em aproximadamente $40 \%$. Sabe-se que o tratamento com a utilização da drotrecogina alfa (ativada) ou proteína $C$ ativada humana recombinante inicialmente apresentou bons resultados na sobrevivência da sepse, porém não houve confirmação de eficácia do tratamento com ela em ensaios, o que levou ao seu desuso e consequente retirada da forma comercial (Xigris) do mercado (ANNANE DJILLALI MD, et al., 2018).

Além disso, o uso de corticosteróide é recorrente, pois melhora a função cardiovascular ao restaurar o volume sanguíneo através da elevação da atividade mineralocorticóide, além de aumentar a resistência vascular sistêmica e suprimir inflamação. Todavia, o uso de corticosteróide tem sua relação risco/benefício cada vez mais avaliada, estando ainda em controvérsia (ANNANE DJILLALI MD, et al., 2018).

Em avaliação do uso das drogas mais adequadas, as evidências apontam que o tempo de desmame dos vasopressores na ventilação mecânica foi mais curto com hidrocortisona e Acetato de Fludrocortisona, sendo essas drogas melhores também no quesito maior número de dias vivos, bem como acelerou a resolução da falência de órgãos em adultos sépticos, auxiliando na preservação da função renal (ANNANE DJILLALI MD, et al., 2018).

Na maioria dos casos de LRA, o tratamento da causa subjacente acarreta a recuperação do quadro clínico como um todo. Todavia, há casos em que alguma Terapia de Substituição Renal (TSR) se faz necessária. A 
TSR é um grupo de possíveis tratamentos que visam substituir as funções de filtração de um rim saudável. No entanto, os critérios para o início e a interrupção dessa terapia não são totalmente consolidados (SOLís GR, et al., 2018).

A TSR intermitente foi considerada, durante anos, o tratamento padrão para pacientes com LRA. Contudo, diante da necessidade de realizar um gerenciamento de fluxos sanguíneos mais altos, essa forma tende a gerar instabilidade hemodinâmica. Diante disso, para tratar um paciente em choque foi desenvolvida a TSR contínua, que consiste em uma técnica extracorpórea de substituição da função renal, objetivando a filtragem de solutos e fluidos do meio intravascular de forma lenta e contínua. Hoje, essa é a modalidade mais utilizada na terapia intensiva e é embasada nos princípios da difusão, ultrafiltração, convecção e adsorção (SOLís GR, et al., 2018).

A TSR inclui diversas modalidades, como a ultrafiltração lenta contínua; hemodiafiltração venovenosa contínua; hemofiltração venovenosa; hemodiálise venovenosa contínua; e hemodiálise venovenosa contínua de alto fluxo. A decisão do melhor momento para finalizar ou alterar a modalidade de tratamento leva em consideração os recursos institucionais locais e a influência de vários fatores como características clínicas do paciente e de seu estado, e características logísticas - disponibilidade de profissionais, custos para a instituição, coagulação do filtro, entre outras. Diante disso, há uma necessidade de estabelecer um consenso nas diretrizes de interrupção dessa terapia, pois a sua não suspensão está associada a altas taxas de mortalidade (SOLíS GR, et al., 2018).

Atualmente, parâmetros como elevação no débito urinário ou redução da creatinina sérica são levados em consideração para suspender a terapia de substituição renal, visto que a oligúria apresenta-se como um marcador para desfechos desfavoráveis nos pacientes com LRA. O teste de estresse com a furosemida é utilizado para avaliação prognóstica em pacientes com o suporte renal suspenso. (SOLís GR, et al., 2018).

A furosemida é um diurético secretado nos túbulos proximais e eliminado em sua maior parte pelos rins. Doses elevadas de furosemida intravenosa podem ser utilizadas como uma possibilidade de converter pacientes oligúricos em não oligúricos para, assim, melhorar o prognóstico. Logo, o uso da furosemida nos pacientes com LRA auxilia na previsão de quais pacientes irão desenvolver lesão renal permanente, de acordo com a resposta à administração de diuréticos, sendo que o mesmo pode ser usado para prever a boa recuperação da função renal pós TRS nos pacientes críticos (SOLíS GR, et al., 2018).

Sabe-se que o uso de TSR teve maior ocorrência nos pacientes com sepse, se comparado com os não sépticos, devido a sua superioridade no manejo de pacientes hemodinamicamente instáveis, além de oferecer baixa remoção de solutos de forma contínua, sendo que a forma da terapia utilizada mais frequentemente em pacientes sépticos foi a hemofiltração durante os quatro primeiros dias, e em pacientes não sépticos foi a hemodiálise (LUFT J, et al., 2016; PETERS E, et al., 2018).

Segundo estudos realizados nos últimos 10 anos, a incidência de LRA e, consequentemente, a mortalidade devida a essa fatalidade, aumentou drasticamente. Isso se deve não só ao fato das suas complicações, mas também por ainda não haver um tratamento eficaz para tal, sendo assim, imprescindível a detecção precoce (ALOBAIDI R, et al., 2015).

\section{CONSIDERAÇÕES FINAIS}

Pode-se concluir que a LRA é uma importante causa de mortalidade em pacientes em tratamento intensivo. Ela afeta o organismo não apenas a nível renal, mas de forma sistêmica, causando prejuízos a vários outros órgãos. Por apresentar difícil manejo e pela alta taxa de mortalidade associada, torna-se imprescindível a identificação de pacientes com maior risco de desenvolver LRA, visando estabelecer medidas preventivas. Além disso, é essencial diagnosticar a LRA precocemente, visando fornecer uma terapia de manutenção e evitar uma futura intervenção mais invasiva. O manejo do fator desencadeante é fundamental para o sucesso do tratamento, entretanto em alguns casos, principalmente aqueles em que 0 paciente encontra-se hemodinamicamente instável, a TSR se faz necessária. 


\section{REFERÊNCIAS}

1. ALOBAIDI R, et al. Sepsis-Associated Acute Kidney Injury. Seminars in Nephrology, 2015; 35(1): 2-11.

2. AMORIM F, et al. Principais causas para o desenvolvimento de lesão renal aguda em pacientes internados em unidade de terapia intensiva: revisão integrativa. Salusvita, 2017; 36(2): 615-628.

3. ANNANE DJILLALI MD, et al. Hydrocortisone plus Fludrocortisone for Adults with Septic Shock. The New England Journal of Medicine, 2018; 378: 809-818.

4. AZEVEDO LCP, et al. Medicina intensiva: abordagem prática. 4 ed. São Paulo: Editora Manole, 2020; $1216 \mathrm{p}$.

5. BELLOMO R, et al. Acute kidney injury in sepsis. Intensive Care Med, 2017; 43: 816-828.

6. BENICHEL C, et al. Fatores de risco para lesão renal aguda em pacientes clínicos intensivos. Portal Revista de Enfermagem, Botucatu, 2020; 33: 1-11.

7. CHEN X, et al. Early outcome of early-goal directed therapy for patients with sepsis or septic shock: a systematic review and meta-analysis of randomized controlled trials. Clinical Research Paper, 2017; 8: 27510-27519.

8. COSTA NA, et al. Evaluation of peptidylarginine deiminase 4 and PADI4 polymorphisms in sepsis-induced acute kidney injury. Revista da Associação Médica Brasileira, 2020; 66: 2-10.

9. FANI F, et al. Recent advances in the pathogenetic mechanisms of sepsis-associated acute kidney injury. Journal of Nephrology, 2018; 31(3): 351-359.

10. FERREIRA MCR, LIMAB EQ. Impact of the development of acute kidney injury on patients admitted to the pediatric intensive care unit. Jornal de Pediatria, 2020; 96(5): 01-07.

11. HUANG CY, et al. Modelos de predição clínica para lesão renal aguda na unidade de terapia intensiva: uma revisão sistemática. Revista Brasileira de Terapia Intensiva, 2020; 32(01): 1-10.

12. JOANNIDIS M, et al. Prevention of acute kidney injury and protection of renal function in the intensive care unit: update 2017. Conference Reports and Expert Panel, 2017; 43: 730-749.

13. KDIGO. Kidney Disease: Improving Global Outcomes (KDIGO) Acute Kidney Injury Work Group. KDIGO Clinical Practice Guideline for Acute Kidney Injury. Kidney inter., Suppl. 2012; 138p.

14. LUFT J, et al. Lesão renal aguda em unidade de tratamento intensivo: características clínicas e desfechos. Cogitare Enferm., 2016; 21(2): 01-09.

15. MAKRIS K, SPANOU L. Acute Kidney Injury: Definition, Pathophysiology and Clinical Phenotypes. The Clinical Biochemist Reviews, 2016; 37(2): 85-98.

16. MARIK PE, et al. Hydrocortisone, Vitamin C, and Thiamine for the Treatment of Severe Sepsis and Septic Shock: A Retrospective Before-After Study. Official Publication of the American College of Chest Physicians, 2017; 15: 12291238.

17. MARTÍNEZ-GARCÍAA JJ, et al. Balance de líquidos y lesión renal aguda en el choque séptico. Boletín Médico del Hospital Infantil de México, 2017; 74(4): 282-288.

18. PETERS E, et al. A worldwide multicentre evaluation of the influence of deterioration or improvement of acute kidney injury on clinical outcome in critically ill patients with and without sepsis at ICU admission: results from The Intensive Care Over Nations audit. Critical Care, 2018; 188.

19. PINHEIRO KHE, et al. Fatores de risco e mortalidade dos pacientes com sepse, lesão renal aguda séptica e não séptica na UTI. Braz. J. Nephrol. (J. Bras. Nefrol), 2019; 41(4): 462-471.

20. SANTOS RP, et al. "An epidemiologic overview of acute kidney injury in intensive care units." Revista da Associação Médica Brasileira (1992), 2019; 65(8): 1094-1101.

21. SANTOS RP, et al. Non-recovery of renal function is a strong independent risk factor associated with mortality in AKI patients. Brazilian Journal of Nephrology, 2020; 42(3): 290-299.

22. SILVA KGN, et al. Influência das variações da pressão positiva sobre a função renal. REME, 2020; 24(1287): 1-5.

23. SKUBE SJ, et al. Acute Kidney Injury and Sepsis. Surgical infections, 2018; 19(2): 216-224.

24. SOLís GR, et al. Prueba de estrés con furosemida para predecir el éxito o fracaso del retiro de la terapia de reemplazo renal lenta continua en lesión renal aguda. Medicina crítica (Colegio Mexicano de Medicina Crítica), 2018; 32(2): 85 92.

25. THONGPRAYOON C, et al. Diagnostics, Risk Factors, Treatment and Outcomes of Acute Kidney Injury in a New Paradigm. Journal of Clinical Medicine, 2020; 9(4): 1-21.

26. YOUNES-IBRAHIM M, et al. The kidney: function, cells and biomarkers. Brazilian Journal of Nephrology., ahead of print Epub, 2021; $1-2$. 